\title{
Procyanidins alleviates morphine tolerance by inhibiting activation of NLRP3 inflammasome in microglia
}

Yang $\mathrm{Cai}^{1+}{ }^{1+}$ Hong Kong ${ }^{1 \dagger}$, Yin-Bing Pan ${ }^{2+}$, Lai Jiang ${ }^{1}$, Xiu-Xiu Pan ${ }^{1}$, Liang Hu${ }^{1}$, Yan-Ning Qian ${ }^{2}$, Chun-Yi Jiang ${ }^{1 *}$ and Wen-Tao Liu ${ }^{1 *}$

\begin{abstract}
Background: The development of antinociceptive tolerance following repetitive administration of opioid analgesics significantly hinders their clinical use. Evidence has accumulated indicating that microglia within the spinal cord plays a critical role in morphine tolerance. The inhibitor of microglia is effective to attenuate the tolerance; however, the mechanism is not fully understood. Our present study investigated the effects and possible mechanism of a natural product procyanidins in improving morphine tolerance via its specific inhibition on NOD-like receptor protein3 (NLRP3) inflammasome in microglia.
\end{abstract}

Methods: CD-1 mice were used for tail-flick test to evaluate the degree of pain. The microglial cell line BV-2 was used to investigate the effects and the mechanism of procyanidins. Reactive oxygen species (ROS) produced from BV-2 cells was evaluated by flow cytometry. Cell signaling was measured by western blot assay and immunofluorescence assay.

Results: Co-administration of procyanidins with morphine potentiated its antinociception effect and attenuated the development of acute and chronic morphine tolerance. Procyanidins also inhibited morphine-induced increase of interleukin-1 $\beta$ and activation of NOD-like receptor protein3 (NLRP3) inflammasome. Furthermore, procyanidins decreased the phosphorylation of p38 mitogen-activated protein kinase, inhibited the translocation of nuclear factor-KB (NF-KB), and suppressed the level of reactive oxygen species in microglia.

Conclusions: Procyanidins suppresses morphine-induced activation of NLRP3 inflammasome and inflammatory responses in microglia, and thus resulting in significant attenuation of morphine antinociceptive tolerance.

Keywords: Morphine tolerance, Procyanidins, NLRP3 inflammasome, Interleukin-1ß, Microglia

\section{Background}

Morphine as the gold standard for treating pain is widely used in clinic. However, long-term use of morphine leads to analgesic tolerance, which greatly reduces the utilization of this drug. The mechanism of morphine tolerance is complicated involving many aspects, such as receptors, ion channels and neural networks [1-3].

For a long period, most investigations focused morphine tolerance on neurons. A lot of evidences suggest

\footnotetext{
* Correspondence: jcy@njmu.edu.cn; painresearch@njmu.edu.cn †'Equal contributors

${ }^{1}$ Jiangsu Key Laboratory of Neurodegeneration, Department of Pharmacology, Nanjing Medical University, 140 Han-Zhong Road, Nanjing 210029, China

Full list of author information is available at the end of the article
}

that morphine activates opioid receptors, resulting in an endocytosis of $\mu$-opioid receptor (MOR) and upregulated expression of $\mathrm{N}$-methyl-D-aspartic acid (NMDA) receptors in the cell surface [4-7]. The phosphorylated NMDA receptors exhibit an enhanced calcium conductance [8], leading to an upregulation of protein kinase $C$ (PKC) $[6,9,10]$, which potentiates neuronal excitability and finally lead to opiate tolerance.

However, compelling evidences reported recently have shown that morphine tolerance is also due to the activation of microglia [11-13]. Chronic morphine exposure induces persistent activation of spinal cord microglia $[14,15]$. Studies demonstrate that morphine binds to Toll-like receptor 4 (TLR4), leading to the initiation of 
innate immune signaling cascade and the production of proinflammatory factors [16].

The microglia activated by morphine releases proinflammatory cytokines, including interleukin (IL)-1 , tumor necrosis factor (TNF)- , and IL-6 [6]. These cytokines enhance the hyperactivity of dorsal horn neurons, which cause the central sensitization and reduction in morphine analgesic efficacy [17]. As the most important factor in inflammatory processes [18], IL-1 has a unique molecular mechanism for its maturation in an intracellular multiprotein complex named as inflammasome. In microglia, proIL-1 was processed by NOD-like receptor protein3 (NLRP3) inflammasome and then the mature IL-1 was secreted [19].

NLRP3 is a unique inflammasome whose assembly and activation involves a two-step process. Firstly, a priming signal stimulates TLR4 and then enhances NF-

B-driven transcription of NLRP3 [20]. Then a second signal promotes NLRP3 to form a protein complex with apoptosis-associated speck-like protein (ASC) containing a caspase recruitment domain [21-23].

Currently, there are three models of NLRP3 activation to illuminate this process. First, the generation of reactive oxygen species (ROS) is considered to be critical for activation of NLRP3 inflammasome. ROS induces the dissociation of thioredoxin and then activates NLRP3 [24]. Second, extracellular ATP binds to P2X7R and triggers $\mathrm{K}^{+}$efflux, which is a sufficient signal for NLRP3 activation [25-27]. Then, a third model has been proposed that certain particulate activators can activate NLRP3 inflammasome by Cathepsin B released from destabilized lysosomal compartment [28].

The function of NLRP3 inflammasome is crucial for the regulation of neuroinflammation mediated by microglia [19]. Therefore, compounds that inhibit the activation of NLRP3 inflammasome may contribute to the improvement of morphine tolerance. Procyanidins is a potent and safe natural product usually extracted from grape seed. It includes a structurally diverse set of compounds, with potentially hundreds of individual compounds with anti-oxidant, anti-inflammatory, and anti-allergic activities [29-31]. Furthermore, procyanidins is available from a wide variety of dietary sources: grapes, nuts, cocoa, apples, peaches, berries, pears, cranberries, and peanuts. Recently, it was recognized as health protective agent [32, 33]. Epidemiological studies indicated that populations consuming procyanidinsrich foods had lower incidences of inflammatory diseases, including metabolic syndrome and atherosclerosis [34, 35]. There is a large number of scientific evidence manifesting the inhibitory effects of procyanidins on inflammation [36].

Herein, we demonstrate for the first time that morphine can activate microglial NLRP3 inflammasome. Moreover, procyanidins, a safe and effective natural product, can attenuate morphine tolerance by inhibiting the NLRP3 activation of inflammasome and the maturation of IL-1 through the unique and potent efficacy of ROS clearance.

\section{Methods \\ Ethics statement}

All procedures were strictly performed in accordance with the regulations of the ethics committee of the International Association for the Study of Pain and the Guide for the Care and Use of Laboratory Animals (The Ministry of Science and Technology of China, 2006). All animal experiments were approved by the Nanjing Medical University Animal Care and Use Committee, and were designed to minimize suffering and the number of animals used.

\section{Animals}

Adult CD-1 mice (18-22 g) were provided by the Experimental Animal Center at Nanjing Medical University, Nanjing, China. Mice were housed five to six per cage under pathogen-free conditions with soft bedding under controlled temperature $\left(22 \pm 2{ }^{\circ} \mathrm{C}\right)$ and a $12 \mathrm{~h} \mathrm{light/dark}$ cycle (lights on at 8:00 a.m.). Behavioral testing was performed during the light cycle (between 9:00 a.m. and 5:00 p.m.). Mice were allowed to acclimate to these conditions for at least 2 days before inclusion in experiments. For each group of experiments, the animals were matched by age and body weight.

\section{Reagents}

Procyanidins was purchased from Zelang Pharmaceutical Co. Ltd (Nanjing, China). The purity of procyanidins was more than $95 \%$. Procyanidins contained $1.1 \%$ monomeric, $34.2 \%$ dimeric, $24.9 \%$ trimeric, $6.7 \%$ tetrameric (totally $66.9 \%$ oligomeric procyanidins), and $33.1 \%$ polymeric procyanidins. IL-1 was from Santa Cruz Biotechnology (Santa Cruz, CA, USA). Antibodies for caspase-1 and NLRP3 were acquired from Adipogen International (San Diego, CA, USA). Antibody for glyceraldehyde-3-phosphate dehydrogenase (GAPDH) was from Sigma-Aldrich (St. Louis, MO, USA). Antibody for ionized calcium-binding adapter molecule 1 (IBA-1) was from Wako Pure Chemical Industries (Osaka, Japan). The antibodies for p38, c-Jun N-terminal kinase (JNK), extracellular-regulated protein kinases (ERK), NMDA-NR1, PKC, p65/RelA, and immunofluorescent antibody for IBA-1 were from Abcam (Cambridge, MA, USA). Antibodies for phosphorylated NR1 subunit (Ser896), phosphorylated PKC (pan) (gamma Thr514), phosphorylated p38 (Tyr182), phosphorylated ERK (Thr202/Tyr204), phosphorylated JNK (Thr183/ Tyr185) and immunofluorescent antibody for c-fos were from Cell Signaling Technology (Beverly, MA, USA). Immunofluorescent antibody for calcitonin gene related peptide (CGRP) was from Santa Cruz Biotechnology (Santa 
Cruz, CA, USA). Lipopolysaccharide (LPS), rotenone, dimethyl sulfoxide (DMSO), and ATP were purchased from Sigma-Aldrich (St. Louis, MO, USA). Morphine hydrochloride was purchased from Shenyang First Pharmaceutical Factory, Northeast Pharmaceutical Group Company (Shenyang, China). Fetal bovine serum (FBS) was purchased from Gibco, and other cell culture media and supplements were purchased from HyClone (Logan, UT, USA). 3-(4,5-dimethyl-2-thiazolyl)-2,5-diphenyl-2-Htetrazolium bromide (MTT) was purchased from Sunshine Biotechnology (Nanjing, China). MitoSOX was purchased from Thermo Fisher Scientific (Waltham, MA, USA). All other reagents were from Sigma-Aldrich (St. Louis, MO, USA).

\section{Cell preparation and stimulation}

BV-2 cells were maintained in humidified $5 \% \mathrm{CO} 2$ at $37^{\circ} \mathrm{C}$ in Dulbecco's modified Eagle's Medium (DMEM) supplemented with $10 \%(v / v)$ FBS, penicillin $(100 \mathrm{U} / \mathrm{ml})$, and streptomycin $(100 \mathrm{U} / \mathrm{ml})$. For inducing inflammasome activation, $10^{5}$ cells were plated in 6-well plate overnight and the medium were changed to serum-free medium in the following morning and then the cells were treated with morphine $(200 \mu \mathrm{M})$ or LPS $(1 \mu \mathrm{g} / \mathrm{ml})$ with or without procyanidins ( $1 \%$ DMSO) for $12 \mathrm{~h}$. We performed the DMSO-only as the control. After that, the BV-2 cells were stimulated with ATP $(5 \mathrm{mM})$ for $1 \mathrm{~h}$. Cell extracts and precipitated supernatants were analyzed by immunoblotting.

\section{Tolerance models and behavioral analysis}

We habituated animals in the testing environments for 2 days and carried out behavioral testing in a blinded manner. For the test of chronic tolerance, mice were injected with saline or morphine $(10 \mathrm{mg} / \mathrm{kg})$ subcutaneously every $12 \mathrm{~h}$ for 7 days and analgesia was assessed $0.5 \mathrm{~h}$ after morphine administration by the tail-flick assay every morning [15]. Briefly, mice's tails were placed in $55^{\circ} \mathrm{C}$ hot water, and the latency of tail withdrawal was measured. A cut-off time of $30 \mathrm{~s}$ was set to avoid tissue damage. Procyanidins was suspended in $0.5 \%$ sodium carboxymethyl cellulose (CMC$\mathrm{Na})$. Procyanidins $(20,40$, or $80 \mathrm{mg} / \mathrm{kg})$ was administered intragastrically 15 min before morphine given twice a day from day 1 to day 7 .

\section{Measurement of cytokines}

Mouse IL-1 in supernatants from cell culture were determined using the ELISA kits (R\&D Systems, Minneapolis, MN, USA) according to manufacturer's instructions. Level of malondialdehyde (MDA) was assayed with kits from Nanjing Jiancheng Bioengineering Institute (Nanjing, China) according to manufacturer's instructions.

\section{NF-KB activation assay}

BV-2 cells were plated in class bottom cell culture dishes and treated with morphine $(200 \mu \mathrm{M})$ for $4 \mathrm{~h}$ with or without procyanidins $(100 \mu \mathrm{M})$. Then BV-2 cells were fixed with ice-cold methanol and were permeabilized with $0.25 \%$ Triton X-100/PBST. After blocking with $1 \%$ bovine serum albumin (BSA) in PBST for $1 \mathrm{~h}$, the coverslips with BV-2 cells were incubated for $2 \mathrm{~h}$ at room temperature with the p65/RelA antibody diluted in $1 \%$ BSA (1:50). Then the coverslips were exposed to the fluorescein isothiocyanate (FITC)-conjugated anti-rabbit IgG (1:100, at room temperature for $1 \mathrm{~h})$ and then were rinsed three times with PBS. Finally, the coverslips were stained with $1 \mu \mathrm{g} / \mathrm{mL}$ DAPI (4 ,6-diamidino-2-phenylindole, a fluorescent DNA dye to mark nucleus) for $1 \mathrm{~min}$. Confocal microscopy analyze was carried out using Olympus FV1000 confocal system.

\section{ROS measurement}

BV-2 cells were plated in non-tissue-culture-treated sixwell dishes and stimulated with morphine $(200 \mu \mathrm{M})$ for $12 \mathrm{~h}$ with or without the pre-treatment of procyanidins $(100 \mu \mathrm{M})$. A positive control was stimulated with rotenone $(10 \mu \mathrm{M})$ for $6 \mathrm{~h}$. After the cultivation, supernatant was removed and cells were washed with PBS. Then the cells were incubated with MitoSOX (to measure mitochondriaassociated ROS levels) at $2.5 \mu \mathrm{M}$ in serum-free DMEM for $0.5 \mathrm{~h}$ at $37^{\circ} \mathrm{C}$. After that, cells were washed with warm PBS, removed from plates with cold PBS containing $1 \%$ FBS, and subjected to fluorescence-activated cell sorting (FACS) analysis (Miltenyi MACSQuant Analyzer 10, Germany). The data were analyzed using FlowJo statistical software (Emerald Biotech Co., Ltd).

\section{Western blot}

Samples (cells or spinal cord tissue segments at L1-L6) were collected and washed with ice-cold PBS before being lysed in radio immunoprecipitation assay (RIPA) lysis buffer [Beyotime, Shanghai, China; $50 \mathrm{mmol} / \mathrm{L}$ Tris (pH 7.4), $150 \mathrm{mmol} / \mathrm{L} \mathrm{NaCl}, 1 \%$ Triton X-100, $1 \%$ sodium deoxycholate, $0.1 \%$ sodium dodecyl sulfate, $1 \mathrm{mmol} / \mathrm{L}$ phenylmethylsulfonyl fluoride, $0.15 \mathrm{U} / \mathrm{mL}$ aprotinin, and $1 \mathrm{mg} / \mathrm{mL}$ pepstatin] and then sample lysates were separated by SDS-PAGE and electrophoretically transferred onto polyvinylidene fluoride membranes (Millipore Corp., Bedford, MA, USA). The membranes were blocked with $10 \%$ whole milk in TBST (Tris- $\mathrm{HCl}$, $\mathrm{NaCl}$, Tween 20) for $2 \mathrm{~h}$ at room temperature, probed with primary antibodies at $4{ }^{\circ} \mathrm{C}$ overnight [GAPDH, 1:8000; IBA-1, 1:1000; NLRP3, 1;1000; caspase-1, 1:1000; IL-1 , 1:500; TNF- , 1:1000; p-p38 (Tyr182), 1:1000; p38, 1:1000; p-ERK, 1:1000; ERK, 1:1000; p-JNK, 1:1000; JNK, 1:1000; p-NR1(Ser896), 1:1000; NR1，1:1000; p-PKC, 1:1000; PKC, 1:1000;] and then incubated with horseradish 
peroxidase-coupled secondary antibodies from Cell Signaling Technology (Beverly, MA, USA). Data were acquired with the Molecular Imager (Gel DocTM XR, 170-8170) and analyzed with Quantity One-4.6.5 (BioRad Laboratories, Berkeley, CA, USA).
Immunofluorescence assay

Under deep anesthesia by intraperitoneal injection of sodium pentobarbital $(100 \mathrm{mg} / \mathrm{kg})$, the animal was perfused with normal saline followed by $4 \%$ paraformaldehyde in 0.1 M PBS, pH 7.2-7.4, for $20 \mathrm{~min}$. Then L4 and/or L5






\section{(See figure on previous page.)}

Fig. 1 Procyanidins suppressed acute and chronic morphine tolerance via inhibiting microglia in the spinal cord. Tail-flick method was performed to evaluate the effect of procyanidins on the morphine tolerance. Data $(n=8)$ were shown as percentage of maximal possible effect (\% MPE). a Procyanidins suppressed acute morphine tolerance. Before the treatment of morphine (10 mg/kg, s.c.), mice were pre-treated with different doses of procyanidins (i.g.) for $15 \mathrm{~min}$. MPE was measured after morphine $10 \mathrm{mg} / \mathrm{kg}$ (s.c.) administration at the first day. b Procyanidins suppressed chronic morphine tolerance. Morphine was injected subcutaneously twice a day, and the MPE was measured $0.5 \mathrm{~h}$ after the first injection of each day. Before the treatment of morphine (10 mg/kg, s.c.), mice were pre-treated with different doses of procyanidins (i.g.) for 15 min. c Procyanidins inhibited morphine-induced upregulation of IBA-1 and phosphorylation of p38 MAPK, but not the p38 total protein. Samples were obtained and analyzed $2 \mathrm{~h}$ after morphine treatment on day 7. Representative western blot data $(n=4)$ for p-p38, p38, and IBA-1 was shown. $\mathbf{d}$ Immunofluorescence images and analysis showed the activation of microglia after morphine injection $(n=4)$ in the spinal cord. ${ }^{*} p<0.05,{ }^{* *} p<0.01,{ }^{* *} p<0.001$ vs. baseline; ${ }^{\#} p<0.05$,

${ }^{\# \#} p<0.01$ vs. morphine-treated group. Scale bar $75 \mathrm{~m}$

lumbar segment were dissected out and post-fixed in the same fixative. The embedded blocks were sectioned as $30-\mu \mathrm{m}$ thick and processed for immunofluorescence assay. Sections from each group (five mice in each group) were incubated with primary antibody (IBA-1, 1:200; c-fos, 1:200; CGRP, 1:200; NeuN, 1:300). Then the free-floating sections were washed with PBS, and incubated with the secondary antibody (1:300; Jackson Laboratories, USA) for $2 \mathrm{~h}$ at room temperature. After being washed three times with PBS, the samples were investigated with a confocal microscope (Leica TCS SPEII, Leica Biosystems, Wetzlar, Germany) for morphologic details. Images were randomly coded and transferred to a computer for further analysis.

\section{Statistical analysis}

SPSS Rel 15 (SPSS Inc., Chicago, IL, USA) was used to conduct all the statistical analyses. Data were statistically evaluated by two-way analysis of variance (ANOVA) followed by Bonferroni post hoc tests. The mean fluorescent pixels of IBA-1 and CGRP were measured by Image Pro Plus 6.0 (Media Cybernetics, Silver Spring, MD, USA). Results were represented as mean \pm standard error of the three independent experiments. Results described as significant were based on a criterion of $p<0.05$.

\section{Results}

Systemic administration of procyanidins improved the morphine tolerance and suppressed morphine-induced microglial activation in the spinal cord

Some experiments were performed to investigate the role of procyanidins on morphine-induced tolerance in vivo. Procyanidins did not alter the pain threshold of mice up to $80 \mathrm{mg} / \mathrm{kg}$. Data suggested that procyanidins significantly improved acute and chronic morphine tolerance (Fig. 1a, b). The MPE decreased to $16.52 \%$ in chronically morphine-treated mice on day 7 . The reduction in morphine's MPE was significantly prevented by twice-daily coadministration of procyanidins $(20,40$, or $80 \mathrm{mg} / \mathrm{kg}$, i.g.). MPE decreased to 57.76, 72.88, and $46.11 \%$, respectively (Fig. 1b).

Subcutaneous injection of morphine induced activation of microglia in the spinal cord. Western blot and immunofluorescence data showed that repeated morphine treatment $(10 \mathrm{mg} / \mathrm{kg}$, twice a day for 7 days $)$ resulted in an upregulation of the IBA-1 (a microglial marker) (Fig. 1c, d). Systemic administration of procyanidins inhibited morphine-induced microglial activation. Strikingly, $40 \mathrm{mg} / \mathrm{kg}$ procyanidins nearly reversed the increase of IBA-1 (Fig. 1c, d). Procyanidins also significantly reduced the upregulation of p38 MAPK phosphorylation induced by morphine exposure in the spinal cord (Fig. 1c). These data provided additional evidences that procyanidins could inhibit morphineinduced microglia activation.

\section{Procyanidins inhibited morphine-induced NLRP3 inflammasome activation in vivo}

It was reported that morphine tolerance led to increased inflammatory response, especially the upregulation of IL1 [6, 37]. Our data showed that consistent morphine exposure $(10 \mathrm{mg} / \mathrm{kg}$, twice a day for 7 days) increased protein levels of the proinflammatory cytokines IL-1 and TNF- in mouse spinal cords. The repetitive administration of procyanidins (i.g., 15 min before each injection of morphine daily) suppressed the upregulation of proinflammatory cytokines in an efficient manner (Fig. 2a, d). IL-1 was processed by NLRP3 inflammasome in microglia [38]. Our data suggested that morphine exposure activated NLRP3, and procyanidins could inhibit this activation (Fig. 2c). Western blot analysis revealed that procyanidins could significantly reduce the increased protein levels of the caspase-1 (Fig. 2b) and the NLRP3, which played a well-established role in neuroinflammation.

MDA level can indirectly reflect attack severity of the body by ROS. Treatment of mice with morphine $(10 \mathrm{mg} / \mathrm{kg}$, twice a day for 7 days) caused an increase of the MDA level in the spinal cord, while pretreatment with procyanidins markedly attenuated the increasing level of MDA (Fig. 2e).

\section{Procyanidins attenuated chronic morphine tolerance by inhibiting the phosphorylation of NMDA-NR1, PKC, and MAPKs in the spinal cord}

Studies show that activated microglia released proinflammatory cytokines, such as IL-1 and TNF- , which 





(See figure on previous page.)

Fig. 2 Procyanidins suppressed morphine-induced NLRP3 inflammasome activation in the spinal cord. a Procyanidins inhibited the morphine-induced upregulation of IL-1 $\beta$ in the spinal cord. $\mathbf{b}$ Procyanidins suppressed the morphine-induced activation of caspase-1 in the spinal cord. $\mathbf{c}$ Procyanidins inhibited the morphine-induced upregulation of NLRP3 in the spinal cord. $\mathbf{d}$ Procyanidins inhibited the morphine-induced upregulation of TNF- in the spinal cord. The western blot samples $(n=4)$ were collected as described in methods. e Procyanidins inhibited the morphine-induced upregulation of malondialdehyde (MDA) in the spinal cord $(n=4) .{ }^{*} p<0.05,{ }^{* *} p<0.01$ vs. baseline; ${ }^{\#} p<0.05,{ }^{\# \#} p<0.01$ vs. morphine-treated group

stimulated neuron and glia through IL-1 receptor, NMDA receptor, and TNF receptor, resulting in the phosphorylation of PKC and MAPKs [37]. As a result, morphine tolerance and hyperalgesia were developed. We investigated the role of procyanidins on morphineinduced activation of neuron and glia in vivo. Western blot analysis revealed that procyanidins suppressed the morphine-induced upregulation of phosphorylated NMDAR-NR1 subunit and PKC in the spinal cords (Fig. 3a). Confocal images and immunofluorescence analysis showed that procyanidins could inhibit the activation of neuronal $\mathrm{c}$-fos and CGRP after morphine treatment in the spinal cord (Fig. 3c, d). Furthermore, procyanidins also inhibit the increased phosphorylation of JNK and phosphorylation of ERK after morphine treatment (Fig. 3b).

\section{Procyanidins suppressed morphine-induced NLRP3 inflammasome activation in vitro}

To study the effects of procyanidins on morphineinduced microglial NLRP3 inflammasome activation in vitro, immortalized murine microglial cell line BV-2 was used $[39,40]$. BV-2 cells were treated with morphine $(200 \mu \mathrm{M})$ and different concentrations of procyanidins for $12 \mathrm{~h}$ with or without ATP for $1 \mathrm{~h}$. Morphine treatment triggered the first step of activating NLRP3 inflammasome, which enhancing the level of NLRP3 and pro-IL-1 (Fig. 4a), and activated the second step, leading to the increasing level of IL-1 and caspase-1 in the supernatant (Fig. 4b, c). Compared with the only morphine-treated group, pre-administrated group with procyanidins $(100 \mu \mathrm{M}) 15$ min before morphine administration significantly reduced the level of pro-IL-1 , IL-1 , NLRP3, and caspase-1 in BV-2 cells (Fig. 4). Moreover, procyanidins also suppressed the upregulation of NLRP3 inflammasome caused by classical TLR4 agonist LPS (Fig. 4) in microglia.

The p38 MAPK phosphorylation and p65 NF- B translocation from the cytoplasm to the nucleus were shown to promote the maturation of NLRP3 inflammasome as the first activation signals in microglia [37]. Compared with the only morphine-treated group, preadministrated (15 $\mathrm{min}$ ) with procyanidins significantly reduced these effects (Fig. 5a, c). However, in BV-2 cells, procyanidins $(100 \mu \mathrm{M})$ alone showed no significant effects on the phosphorylation of p38 MAPK and the translocation of p65 NF- B (Fig. 5a, c). MTT assay indicated that the different doses of procyanidins did not affect cell proliferation (Fig. 5b).

Procyanidins as a potent free radical scavenger has demonstrated the ability to clear intracellular ROS. The main source of cellular ROS is mitochondria [41]. Various stresses including increased metabolic rates, hypoxia, or inflammatory response could markedly increase the production of mitochondrial ROS, which would activate NLRP3 inflammasome as the second activation signal in microglia [38]. To investigate a possible mechanism of morphine in inflammasome activation, we measured ROS production in mitochondria by flow cytometry [42]. Morphine significantly increased the level of ROS compared with the negative and positive control (Fig. 5d). Preadministrated (15 min) with procyanidins $(100 \mu \mathrm{M})$ significantly reduced morphine-induced production of ROS (Fig. 5d) and procyanidins also inhibited the ROS induced by rotenone (Additional file 1: Figure S1).

\section{Discussion}

In this study, we found procyanidins, a clinically used health product, had a significant inhibitory effect on morphine-induced activation of microglia by suppressing NLRP3 inflammasome activation. Therefore, procyanidins attenuated the development of acute and chronic morphine tolerance markedly. This study may provide a new solution to improve clinical analgesic efficacy of opioids through the inhibition of microglial NLRP3 inflammasome.

Procyanidins, as safe and effective compounds, can be absorbed from the gastrointestinal tract when people eat certain fruits and vegetables. It was usually administrated orally $[43,44]$. However, the oral bioavailability of procyanidins was about $3-4 \%$, which was not very satisfactory though it had reached the standard of many clinically used drugs such as levodopa, a drug used to treat Parkinson's disease [44-46]. More fortunately, procyanidins was a relatively safe drug with an oral $\mathrm{LD}_{50}$ value of over $4000 \mathrm{mg} / \mathrm{kg}$. The optimal dose for the attenuation of morphine tolerance is $40 \mathrm{mg} / \mathrm{kg}$ in mice, which equals to $3.33 \mathrm{mg} / \mathrm{kg}$ in human. Therefore, human with a body weight of $60 \mathrm{~kg}$ might intake $400 \mathrm{mg}$ of procyanidins every day if it is given twice a day. At present, a lot of commercial nutrition supplements containing procyanidins recommend a dosage from 400 to $800 \mathrm{mg}$ every day. Therefore, procyanidins would be safe and effective for inhibiting morphine tolerance. These results 


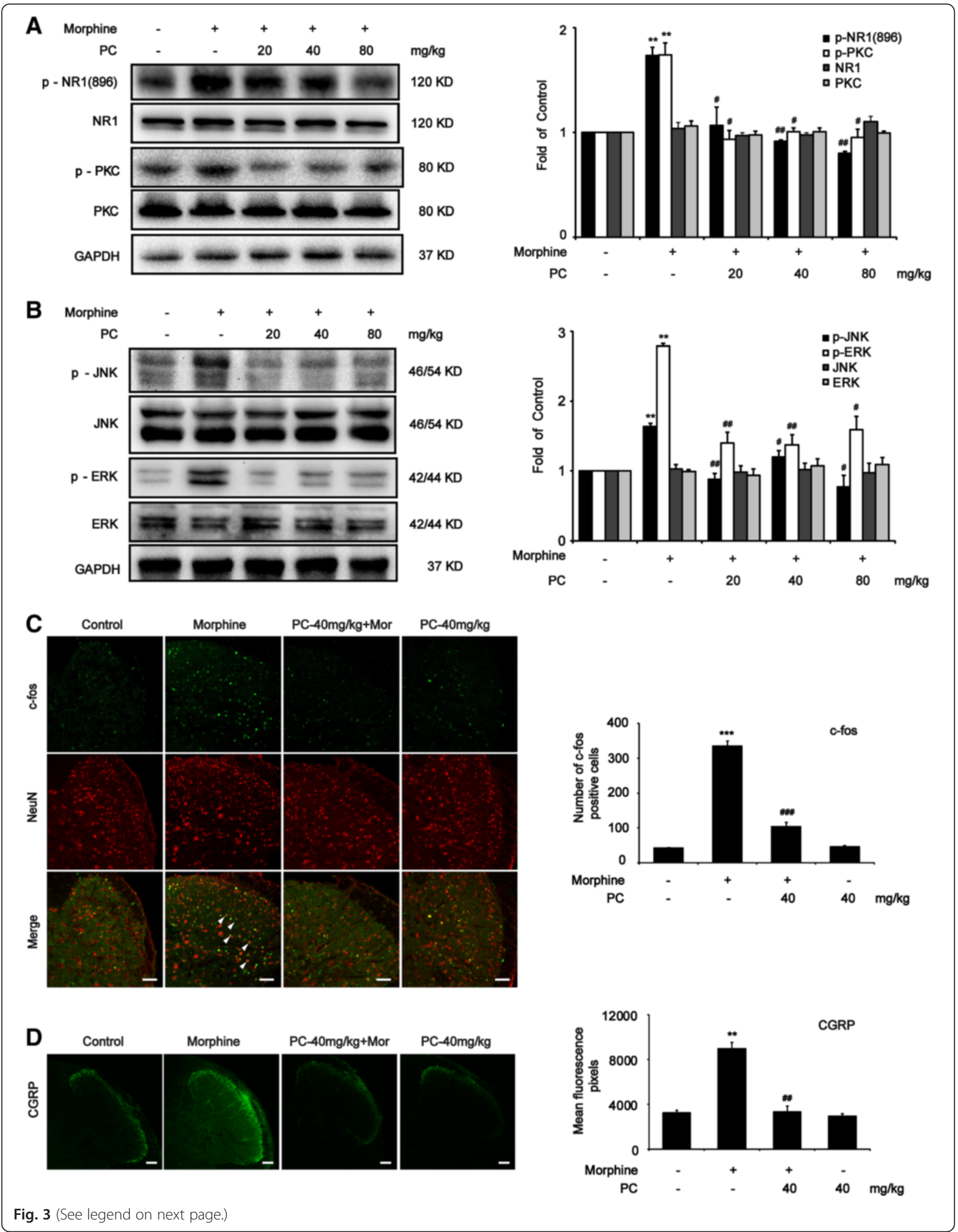


(See figure on previous page.)

Fig. 3 Procyanidins attenuated chronic morphine tolerance by inhibiting the neuron-glia pathway in the spinal cord. a Procyanidins inhibited the morphine-induced upregulation of phosphorylated PKC and phosphorylated NMDAR-NR1 in the spinal cord. The western blot samples $(n=4)$ were collected $2 \mathrm{~h}$ after the last morphine treatment. b Procyanidins inhibited the morphine-induced phosphorylation of JNK and ERK in the spinal cord. The western blot samples $(n=4)$ were collected as above. $\mathbf{c}, \mathbf{d}$ Confocal images and immunofluorescence analysis showed the activation of neuronal $c$-fos and CGRP after morphine treatment $(n=4)$ in the spinal cord. The quantification of c-fos and CGRP immunofluorescence was respectively represented as number of c-fos positive cells and mean fluorescence pixels in the superficial dorsal horns. ${ }^{*} p<0.05,{ }^{* *} p<0.01,{ }^{* * *} p<0.001$ vs. baseline; ${ }^{*} p<0.05$, ${ }^{\# \#} p<0.01,{ }^{\# \#} p<0.001$ vs. morphine-treated group. Scale bar $75 \mathrm{~m}$

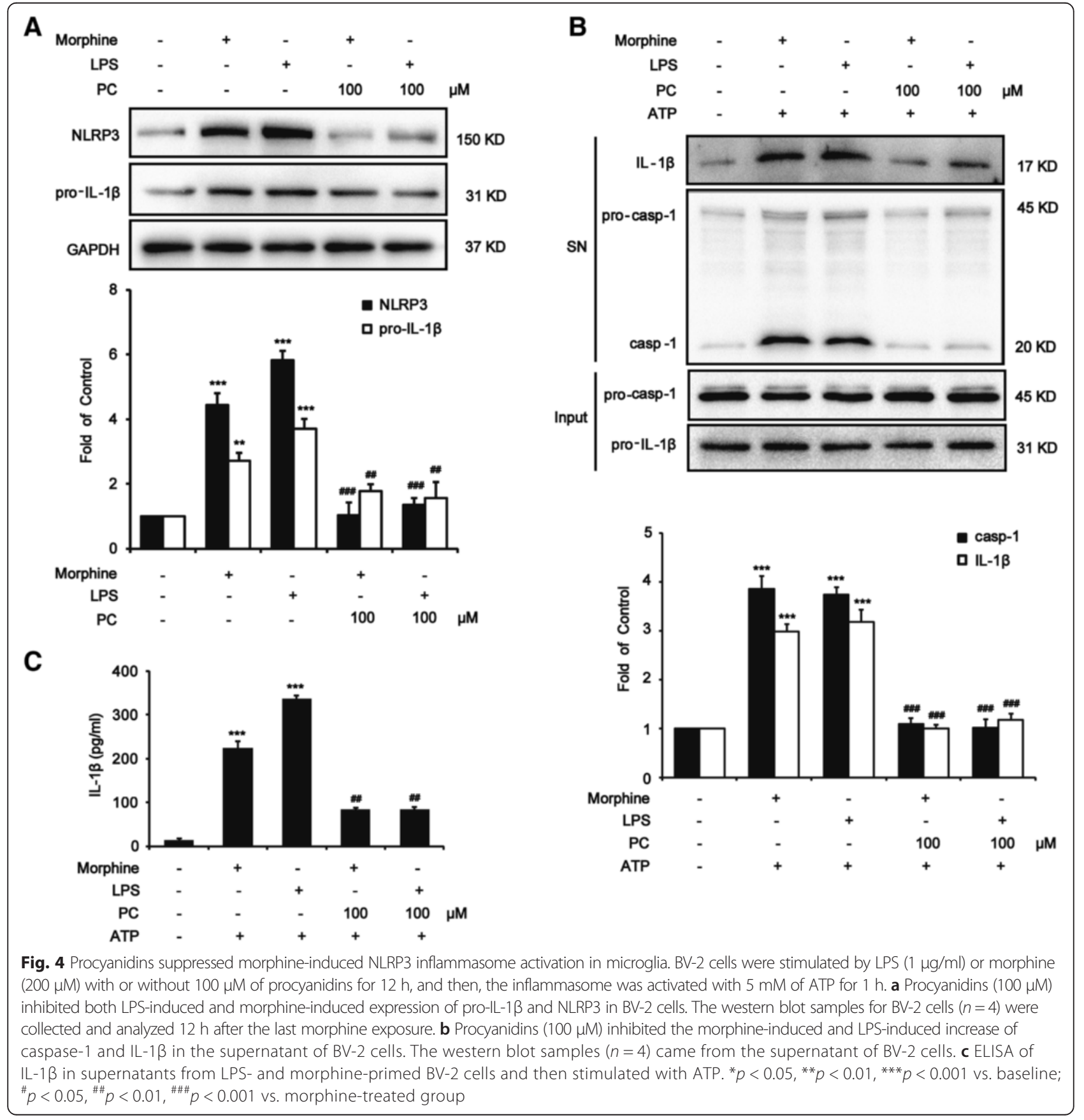




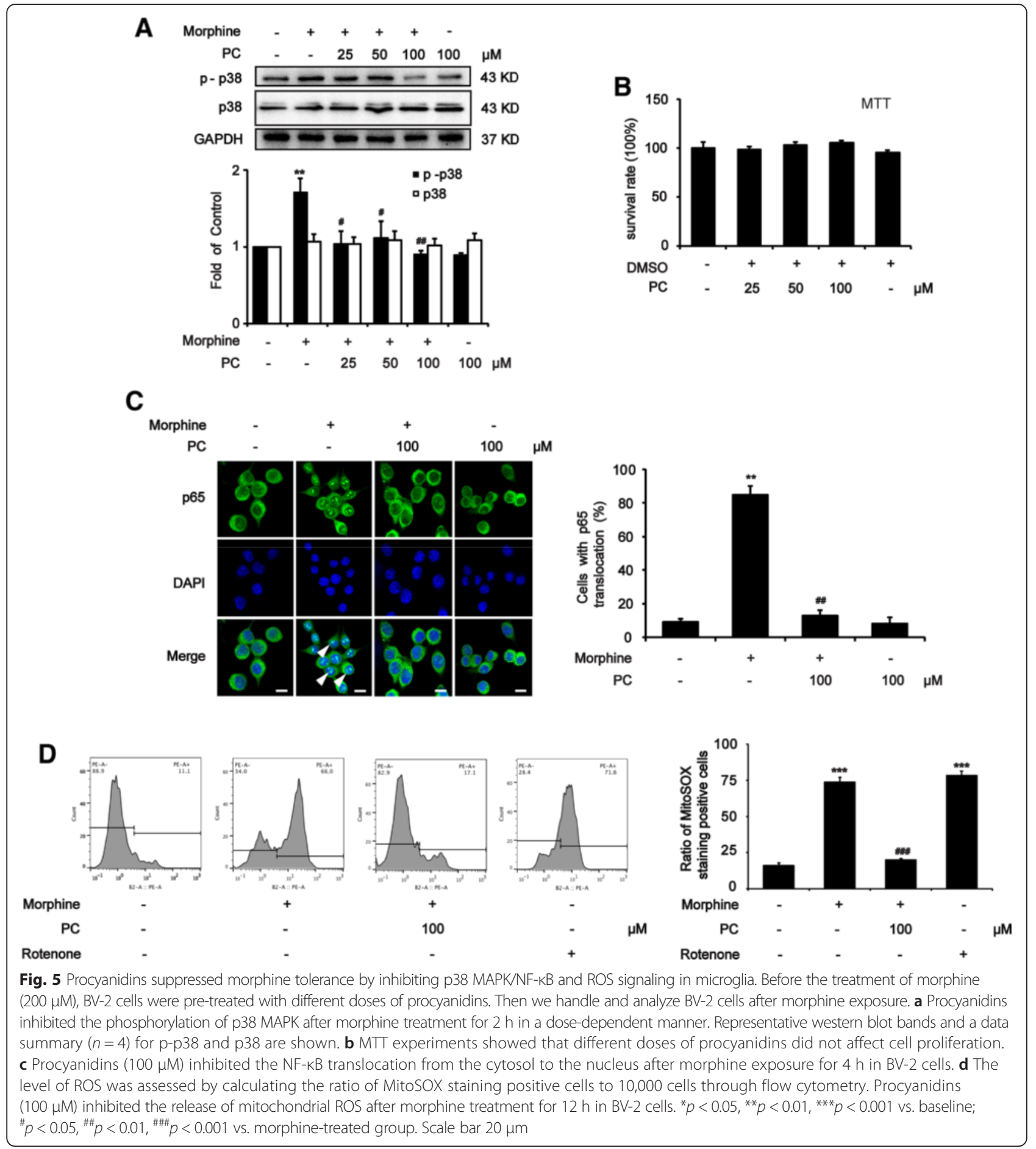

indicate that it may be beneficial for the patients treated with opiate drugs to actively intake foods rich in procyanidins, such as: cranberry, blueberry, apple, cherry, and grape.

In the spinal cord, microglia regulated morphine tolerance via TLR4 signaling [47]. When morphine bound with TLR4, it activated the downstream intracellular signaling pathways similar to those activated by IL-1 binding to its cognate receptor, resulting in a potent proinflammatory signal (Fig. 6) $[6,48]$. Our data showed that, pre-treatment of procyanidins significantly suppressed the level of IL-1 in vivo and in vitro after morphine exposure. Beside of this, adiministration of procyanidins alone did not affect the level of proinflammatory cytokines (Additional file: 1 Figure S2). In microglia, the NLRP3 inflammasome is a molecular platform 


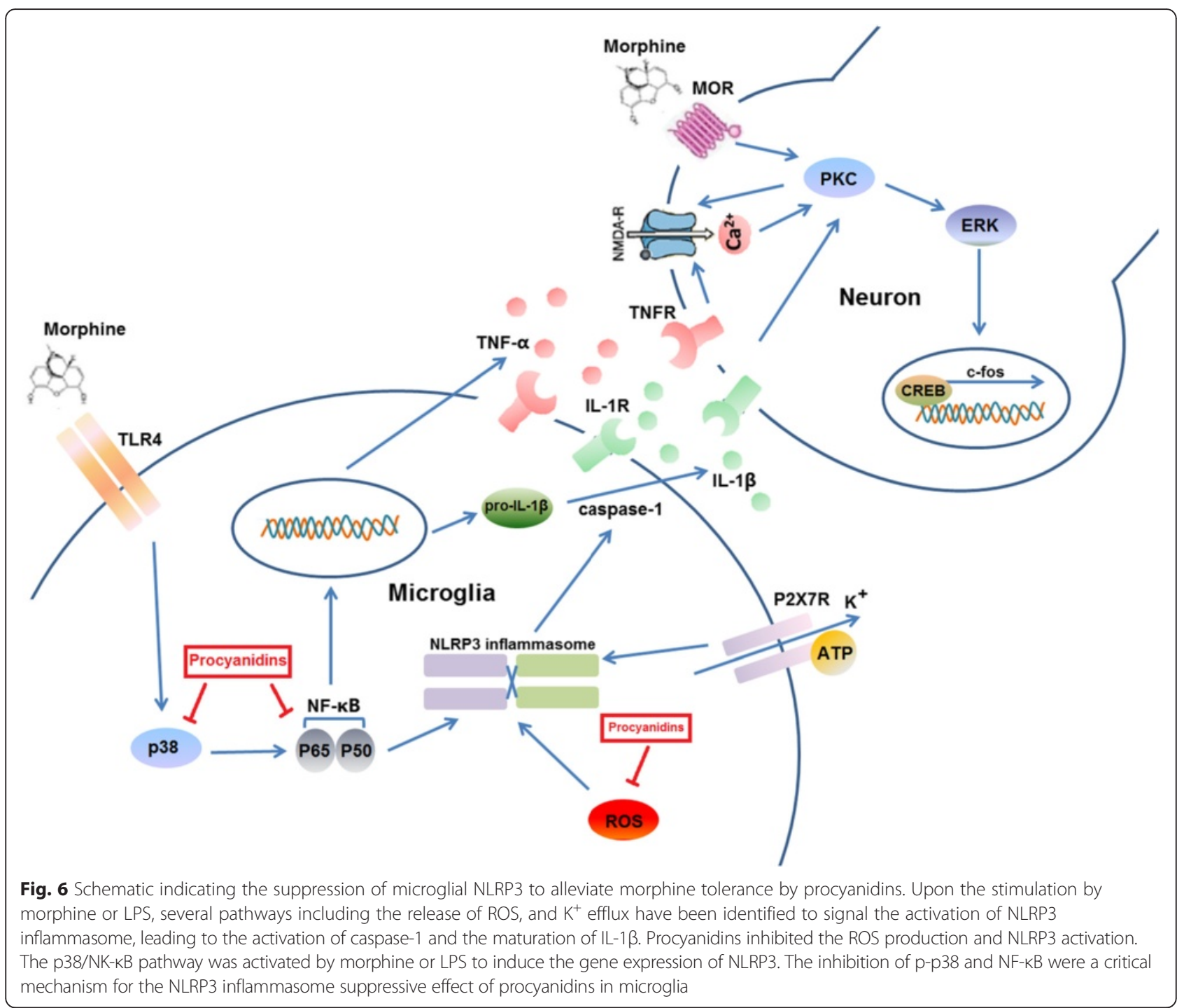

activated upon signals of cellular danger to trigger innate immune defenses through the maturation of proinflammatory cytokines such as IL-1 [38]. We found that procyanidins could inhibit morphine-induced activation of NLRP3 inflammasome in vitro in the concentration of $100 \mu \mathrm{M}$. Though this concentration seems a little high, it could be achievable in vivo. Since the oral bioavailability of procyanidins was about 3-4\% [44-46], the blood concentration of $100 \mu \mathrm{M}$ might be easily achieved when $80 \mathrm{mg} / \mathrm{kg}$ of procyanidins was given to mice which had a blood volume of $1.5 \mathrm{ml}$. However, further investigations are needed into the preclinical pharmacokinetic evaluation of procyanidins, especially on its distribution in the central nervous system.

Some studies reported that spinal P2X7R was involved in the induction but not maintenance of morphine tolerance [27]. As previously mentioned, the activation of P2X7R was viewed as the second signal for the activation of NLRP3 inflammasome in microglia (Fig. 6). It was reported that NAC, a ROS inhibitor, decreased IL-1 synthesis via inhibiting the P2X7R activation in murine macrophages [49]. Therefore, we could not rule out that procyanidins inactivated NLRP3 inflammasome by inhibiting P2X7R after morphine exposure in microglia.

Mao et al. reported that LPS-induced upregulation of NLRP3 was suppressed in the morphine-tolerant state, which may be one of the mechanisms involved in morphine-induced immunosuppression [50]. But to our best knowledge, we first confirmed that in the morphinetolerant state, morphine could significantly activate NLRP3 inflammasome in the microglia.

It said before, ROS could induce the dissociation of thioredoxin and then activate NLRP3 inflammasome (Fig. 6). Our compound, procyanidins, as a ROS scavenger, significantly inhibited the morphine-induced release 
of ROS and then suppressed the microglial NLRP3 inflammasome to attenuate morphine tolerance (Fig. 6). Generation of IL-1 requires a priming signal and activating signal. The priming signal could be mediated by pattern recognition receptor (PRRs) such as TLRs, which would activate NF- B (Fig. 6) [51]. The activated NF- B can promote the transcription of NLRP3 (Fig. 6); therefore, we further investigated the effect of procyanidins on NF- B activation. The mammalian NF- B family members can be regulated by upstream diverse signal pathways, such as TLRs, TNF receptors, IL-1 receptors, and MAPKs $[52,53]$. It was confirmed that morphine could activate TLR4, TNF- , and IL-1 in microglia [54]. Furthermore, microglial p38 MAPK was dramatically and primarily activated by chronic morphine exposure in the spinal cord $[27,55,56]$. Indeed, our study revealed procyanidins could sufficiently inhibit morphine-induced NF- B p65 nuclear translocation and remarkably reduced phosphorylation of p38 MAPK.

Morphine tolerance involves many receptors, channels, and processes. In a positive feedback loop, morphine induces activation of microglia releasing a lot of proinflammatory cytokines, which results in an extraexcitation of sensor neurons in the spinal cord [57-59] and decreases the efficiency of morphine analgesia [60]. This process involves the activation of NMDA/ ERK/PKC signaling, which plays an important role in morphine tolerance [6]. The proinflammatory cytokines such as TNF- and IL-1 activate NMDA receptor by phosphorylating PKC followed by a strong activation of ERK (Fig. 6). Then the phosphorylated ERK and PKC can facilitate many channels and receptors such as calcium channel on the cell membrane, resulting in the central sensitization and morphine tolerance (Fig. 6) $[6,61,62]$. In this study, we found that procyanidins notably inhibited the phosphorylation of NMDA, ERK, and PKC in the spinal cord. In addition to ERK and p38, we also found that procyanidins significantly inhibited the phosphorylation of JNK, which involved in the late stage of morphine tolerance $[63,64]$. It indicated that procyanidins might also have significantly inhibitory effect in the late stage of morphine tolerance.

\section{Conclusions}

In summary, procyanidins could extend acute morphine analgesia and attenuate chronic morphine tolerance with a possible biological mechanism of inhibiting neuroinflammation represented by microglia activation. Our results demonstrated that morphine exposure leading to excess production of ROS from mitochondria, and that procyanidins could diminish ROS and consequently inhibit the activation of NLRP3 inflammasome in microglia. Furthermore, procyanidins could suppress the over- activated NF- B and MAPKs signaling, which played important roles in morphine tolerance. Altogether, our studies suggest that procyanidins may be a potential drug candidate to reduce morphine tolerance and to enhance the clinical utility of opioid drugs.

\section{Additional files}

Additional file 1: Figures S1 and S2. BV2 cells were treated with rotenone for $6 \mathrm{~h}$ with or without the pre-treatment of procyanidins. We found that procyanidins could inhibit the upregulation of ROS which induced by rotenone. Additional controls with procyanidins treatment alone did not affect the baseline levels of NLRP3, pro-casp-1, casp-1, pro-IL-1 $\beta, I L-1 \beta$, and TNF- . (DOCX 237 kb)

\section{Abbreviations}

ASC: apoptosis-associated speck-like protein; BSA: bovine serum albumin; CGRP: c-fos and calcitonin gene-related peptide; CMC-Na: sodium carboxymethyl cellulose; DAPI: 4,6-diamidino-2-phenylindole;

DMEM: Dulbecco s modified Eagle s medium; DMSO: dimethyl sulfoxide; ERK: extracellular regulated protein kinases; FACS: fluorescence-activated cell sorting; FBS: fetal bovine serum; FITC: fluorescein isothiocyanate;

GAPDH: glyceraldehyde-3-phosphate dehydrogenase; IBA-1: ionized calcium binding adapter molecule 1; IL-1 $\beta$ : interleukin-1 $\beta$; IL-6: interleukin-6; JNK: c-Jun N-terminal kinase; LPS: lipopolysaccharide; MAPK: mitogen-activated protein kinase; MDA: malondialdehyde; MOR: -opioid receptor; MTT: 3-(4,5-dimethyl-2-thiazolyl)-2,5-diphenyl-2-H-tetrazolium bromide; NAC: $\mathrm{N}$-acetyl-cysteine; NF-kB: nuclear factor-KB; NLRP: NOD-like receptor family pyrin domain-containing protein; NMDA: N-methyl-D-aspartic acid; PBS: phosphate-buffered saline; PC: procyanidins; PKC: protein kinase C; RIPA: radio immunoprecipitation assay; ROS: reactive oxygen specie; SDS-PAGE: sodium dodecyl sulfate-polyacrylamide gel electrophoresis; TLR4: Toll-like receptor 4; TNF- : tumor necrosis factor

\section{Competing interests}

The authors declare that they have no competing interests.

\section{Authors' contributions}

YC, CJ, and WL designed the experiments with the help of HK, and YP performed the experiments, analyzed the results, and drafted the manuscript. $\mathrm{L}$ and XP performed the immunoassays and behavioral measure. YQ performed the Western blotting analysis. LH carried out the cell cultures. WL and $C J$ secured funding for the project and helped with the final version of the manuscript. All authors read and approved the final manuscript.

\section{Acknowledgements}

This work was supported by the National Natural Science Foundation of China (Nos. 81471142, 81202513, and 81171044) and the Postdoctoral Science Foundation of China (No. 2012M521103).

\section{Author details}

${ }^{1}$ Jiangsu Key Laboratory of Neurodegeneration, Department of Pharmacology, Nanjing Medical University, 140 Han-Zhong Road, Nanjing 210029, China. ${ }^{2}$ Department of Anesthesiology, The First Affiliated Hospital of Nanjing Medical University, Nanjing 210029, China.

Received: 3 October 2015 Accepted: 22 February 2016

Published online: 01 March 2016

\section{References}

1. Ji RR. Targeting microglial purinergic signaling to improve morphine analgesia. Pain. 2010;150:377-8

2. Johansson T, Elfverson M, Zhou Q, Nyberg F. Allosteric modulation of the NMDA receptor by neurosteroids in rat brain and the impact of long term morphine administration. Biochem Biophys Res Commun. 2010;401:504-8.

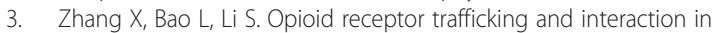
nociceptors. Br J Pharmacol. 2015;172:364-74. 
4. Ogoshi F, Yin HZ, Kuppumbatti Y, Song B, Amindari S, Weiss JH. Tumor necrosis-factor-alpha (TNF-alpha) induces rapid insertion of Ca2+-permeable alpha-amino-3-hydroxyl-5-methyl-4-isoxazole-propionate (AMPA)/kainate (Ca-A/K) channels in a subset of hippocampal pyramidal neurons. Exp Neurol. 2005;193:384-93.

5. Stellwagen D, Beattie EC, Seo JY, Malenka RC. Differential regulation of AMPA receptor and GABA receptor trafficking by tumor necrosis factoralpha. J Neurosci. 2005;25:3219-28.

6. Hutchinson MR, Shavit Y, Grace PM, Rice KC, Maier SF, Watkins LR. Exploring the neuroimmunopharmacology of opioids: an integrative review of mechanisms of central immune signaling and their implications for opioid analgesia. Pharmacol Rev. 2011;63:772-810.

7. Williams JT, Ingram SL, Henderson G, Chavkin C, von Zastrow M, Schulz S, et al. Regulation of mu-opioid receptors: desensitization, phosphorylation, internalization, and tolerance. Pharmacol Rev. 2013;65:223-54.

8. Viviani B, Bartesaghi S, Gardoni F, Vezzani A, Behrens MM, Bartfai T, et al. Interleukin-1beta enhances NMDA receptor-mediated intracellular calcium increase through activation of the Src family of kinases. J Neurosci. 2003;23:8692-700.

9. Morioka N, Inoue A, Hanada T, Kumagai K, Takeda K, Ikoma K, et al. Nitric oxide synergistically potentiates interleukin-1 beta-induced increase of cyclooxygenase-2 mRNA levels, resulting in the facilitation of substance $P$ release from primary afferent neurons: involvement of cGMP-independent mechanisms. Neuropharmacology. 2002;43:868-76.

10. Kleibeuker W, Ledeboer A, Eijkelkamp N, Watkins LR, Maier SF, Zijlstra J, et al. A role for $\mathrm{G}$ protein-coupled receptor kinase 2 in mechanical allodynia. Eur J Neurosci. 2007;25:1696-704.

11. Eidson LN, Murphy AZ. Persistent peripheral inflammation attenuates morphine-induced periaqueductal gray glial cell activation and analgesic tolerance in the male rat. J Pain. 2013:14:393-404.

12. Ferrini F, Trang T, Mattioli TA, Laffray S, Del'Guidice T, Lorenzo LE, et al. Morphine hyperalgesia gated through microglia-mediated disruption of neuronal Cl(-) homeostasis. Nat Neurosci. 2013;16:183-92.

13. Cui Y, Liao XX, Liu W, Guo RX, Wu ZZ, Zhao CM, et al. A novel role of minocycline: attenuating morphine antinociceptive tolerance by inhibition of p38 MAPK in the activated spinal microglia. Brain Behav Immun. 2008;22:114-23.

14. Wen YR, Tan PH, Cheng JK, LiU YC, Ji RR. Microglia: a promising target for treating neuropathic and postoperative pain, and morphine tolerance. J Formos Med Assoc. 2011;110:487-94.

15. Han Y, Jiang C, Tang J, Wang C, Wu P, Zhang G, et al. Resveratrol reduces morphine tolerance by inhibiting microglial activation via AMPK signalling Eur J Pain. 2014;18:1458-70.

16. Hutchinson MR, Lewis SS, Coats BD, Rezvani N, Zhang Y, Wieseler JL, et al. Possible involvement of toll-like receptor 4 /myeloid differentiation factor-2 activity of opioid inactive isomers causes spinal proinflammation and related behavioral consequences. Neuroscience. 2010;167:880-93.

17. Sun J, Liu S, Mata M, Fink DJ, Hao S. Transgene-mediated expression of tumor necrosis factor soluble receptor attenuates morphine tolerance in rats. Gene Ther. 2012;19:101-8.

18. Basu A, Krady JK, Levison SW. Interleukin-1: a master regulator of neuroinflammation. J Neurosci Res. 2004:78:151-6.

19. Gustin A, Kirchmeyer M, Koncina E, Felten P, Losciuto S, Heurtaux T, et al. NLRP3 inflammasome is expressed and functional in mouse brain microglia but not in astrocytes. PLoS One. 2015;10:e0130624

20. Lamkanfi M, Dixit VM. The inflammasomes. PLoS Pathog. 2009;5:e1000510.

21. Davis BK, Wen H, Ting JP. The inflammasome NLRs in immunity, inflammation, and associated diseases. Annu Rev Immunol. 2011;29:707-35.

22. Schroder K, Tschopp J. The inflammasomes. Cell. 2010;140:821-32.

23. Dowling JK, O'Neill LA. Biochemical regulation of the inflammasome. Crit Rev Biochem Mol Biol. 2012;47:424-43.

24. Juliana C, Fernandes-Alnemri T, Kang S, Farias A, Qin F, Alnemri ES. Nontranscriptional priming and deubiquitination regulate NLRP3 inflammasome activation. J Biol Chem. 2012;287:36617-22.

25. Petrilli V, Papin S, Dostert C, Mayor A, Martinon F, Tschopp J. Activation of the NALP3 inflammasome is triggered by low intracellular potassium concentration. Cell Death Differ. 2007;14:1583-9.

26. Di Virgilio F. Liaisons dangereuses: P2X(7) and the inflammasome. Trends Pharmacol Sci. 2007;28:465-72.

27. Zhou D, Chen ML, Zhang YQ, Zhao ZQ. Involvement of spinal microglial P2X7 receptor in generation of tolerance to morphine analgesia in rats. J Neurosci. 2010;30:8042-7.
28. Hornung V, Bauernfeind F, Halle A, Samstad EO, Kono H, Rock KL, et al. Silica crystals and aluminum salts activate the NALP3 inflammasome through phagosomal destabilization. Nat Immunol. 2008;9:847-56.

29. Williams RJ, Spencer JP, Rice-Evans C. Flavonoids: antioxidants or signalling molecules? Free Radic Biol Med. 2004;36:838-49.

30. Rivera L, Moron R, Sanchez M, Zarzuelo A, Galisteo M. Quercetin ameliorates metabolic syndrome and improves the inflammatory status in obese Zucker rats. Obesity (Silver Spring). 2008;16:2081-7.

31. Kim HP, Park H, Son KH, Chang HW, Kang SS. Biochemical pharmacology of biflavonoids: implications for anti-inflammatory action. Arch Pharm Res. 2008;31:265-73.

32. Auger C, Al-Awwadi N, Bornet A, Rouanet J-M, Gasc F, Cros G, et al. Catechins and procyanidins in Mediterranean diets. Food Res Int. 2004;37:233-45.

33. Yilmaz Y, Toledo RT. Health aspects of functional grape seed constituents. Trends Food Sci Technol. 2004;15:422-33.

34. Engler MB, Engler MM. The emerging role of flavonoid-rich cocoa and chocolate in cardiovascular health and disease. Nutr Rev. 2006;64:109-18.

35. Monagas M, Khan N, Andres-Lacueva C, Casas R, Urpi-Sarda M, Llorach R, et al. Effect of cocoa powder on the modulation of inflammatory biomarkers in patients at high risk of cardiovascular disease. Am J Clin Nutr. 2009;90: 1144-50.

36. Fraga CG, Oteiza PI. Dietary flavonoids: role of (-)-epicatechin and related procyanidins in cell signaling. Free Radic Biol Med. 2011;51:813-23.

37. Grace PM, Hutchinson MR, Maier SF, Watkins LR. Pathological pain and the neuroimmune interface. Nat Rev Immunol. 2014;14:217-31.

38. Zhou R, Yazdi AS, Menu P, Tschopp J. A role for mitochondria in NLRP3 inflammasome activation. Nature. 2011:469:221-5.

39. Blasi E, Barluzzi R, Bocchini V, Mazzolla R, Bistoni F. Immortalization of murine microglial cells by a v-raf/v-myc carrying retrovirus. J Neuroimmunol. 1990;27:229-37.

40. Wang X, Loram LC, Ramos K, de Jesus AJ, Thomas J, Cheng K, et al. Morphine activates neuroinflammation in a manner parallel to endotoxin. Proc Natl Acad Sci U S A. 2012;109:6325-30

41. Heid ME, Keyel PA, Kamga C, Shiva S, Watkins SC, Salter RD. Mitochondrial reactive oxygen species induces NLRP3-dependent lysosomal damage and inflammasome activation. J Immunol. 2013;191:5230-8.

42. Brookes PS, Yoon Y, Robotham JL, Anders MW, Sheu SS. Calcium, ATP, and ROS: a mitochondrial love-hate triangle. Am J Physiol Cell Physiol. 2004;287: C817-833.

43. Zhu OY, Schramm DD, Gross HB, Holt RR, Kim SH, Yamaguchi T, et al. Influence of cocoa flavanols and procyanidins on free radical-induced human erythrocyte hemolysis. Clin Dev Immunol. 2005;12:27-34.

44. Del Rio D, Borges G, Crozier A. Berry flavonoids and phenolics: bioavailability and evidence of protective effects. Br J Nutr. 2010;104 Suppl 3:S67-90.

45. Bansode RR, Randolph P, Ahmedna M, Hurley S, Hanner T, Baxter SA, et al. Bioavailability of polyphenols from peanut skin extract associated with plasma lipid lowering function. Food Chem. 2014;148:24-9.

46. Serra A, Macia A, Romero MP, Valls J, Blade C, Arola L, et al. Bioavailability of procyanidin dimers and trimers and matrix food effects in in vitro and in vivo models. Br J Nutr. 2010;103:944-52.

47. Eidson LN, Murphy AZ. Blockade of Toll-like receptor 4 attenuates morphine tolerance and facilitates the pain relieving properties of morphine. $J$ Neurosci. 2013:33:15952-63.

48. O'Neill LA. The interleukin-1 receptor/Toll-like receptor superfamily: 10 years of progress. Immunol Rev. 2008:226:10-8.

49. Zanin RF, Bergamin LS, Morrone FB, Coutinho-Silva R, de Souza Wyse AT, Battastini AM. Pathological concentrations of homocysteine increases IL1 beta production in macrophages in a P2X7, NF-kB, and erk-dependent manner. Purinergic Signal. 2015.

50. Mao X, Sarkar S, Chang SL. Involvement of the NLRP3 inflammasome in the modulation of an LPS-induced inflammatory response during morphine tolerance. Drug Alcohol Depend. 2013;132:38-46.

51. Bryant C, Fitzgerald KA. Molecular mechanisms involved in inflammasome activation. Trends Cell Biol. 2009:19:455-64.

52. Liu J, Wang Y, Ouyang X. Beyond toll-like receptors: porphyromonas gingivalis induces IL-6, IL-8, and VCAM-1 expression through NOD-mediated NF-kappaB and ERK signaling pathways in periodontal fibroblasts. Inflammation. 2014:37:522-33.

53. Yuan S, Zhang J, Zhang L, Huang L, Peng J, Huang S, et al. The archaic roles of the amphioxus NF-kappaB//kappaB complex in innate immune responses. J Immunol. 2013;191:1220-30. 
54. Jiang C, Xu L, Chen L, Han Y, Tang J, Yang Y, et al. Selective suppression of microglial activation by paeoniflorin attenuates morphine tolerance. Eur J Pain. 2015;19:908-19.

55. Cui Y, Chen Y, Zhi JL, Guo RX, Feng JQ, Chen PX. Activation of p38 mitogen-activated protein kinase in spinal microglia mediates morphine antinociceptive tolerance. Brain Res. 2006;1069:235-43.

56. Horvath RJ, Romero-Sandoval EA, De Leo JA. Inhibition of microglial P2X4 receptors attenuates morphine tolerance, Iba1, GFAP and mu opioid receptor protein expression while enhancing perivascular microglial ED2. Pain. 2010;150:401-13.

57. Kawasaki Y, Zhang L, Cheng JK, Ji RR. Cytokine mechanisms of central sensitization: distinct and overlapping role of interleukin-1 beta, interleukin-6, and tumor necrosis factor-alpha in regulating synaptic and neuronal activity in the superficial spinal cord. J Neurosci. 2008;28:5189-94.

58. Zhong Y, Zhou $L$, Ren WJ, Xin WJ, Li YY, Zhang T, et al. The direction of synaptic plasticity mediated by C-fibers in spinal dorsal horn is decided by Src-family kinases in microglia: the role of tumor necrosis factor-alpha. Brain Behav Immun. 2010;24:874-80.

59. Zhang L, Berta T, Xu ZZ, Liu T, Park JY, Ji RR. TNF-alpha contributes to spinal cord synaptic plasticity and inflammatory pain: distinct role of TNF receptor subtypes 1 and 2. Pain. 2011;152:419-27.

60. Matsushita Y, Omotuyi IO, Mukae T, Ueda H. Microglia activation precedes the anti-opioid BDNF and NMDA receptor mechanisms underlying morphine analgesic tolerance. Curr Pharm Des. 2013;19:7355-61.

61. Garzon J, Rodriguez-Munoz M, Sanchez-Blazquez P. Direct association of Muopioid and NMDA glutamate receptors supports their cross-regulation: molecular implications for opioid tolerance. Curr Drug Abuse Rev. 2012;5:199-226.

62. Wang Z, Ma W, Chabot JG, Quirion R. Calcitonin gene-related peptide as a regulator of neuronal CaMKII-CREB, microglial p38-NFkappaB and astroglial ERK-Stat1/3 cascades mediating the development of tolerance to morphine-induced analgesia. Pain. 2010;151:194-205.

63. Marcus DJ, Zee M, Hughes A, Yuill MB, Hohmann AG, Mackie K, et al. Tolerance to the antinociceptive effects of chronic morphine requires $c$-Jun N-terminal kinase. Mol Pain. 2015;11:34.

64. Melief EJ, Miyatake M, Bruchas MR, Chavkin C. Ligand-directed c-Jun Nterminal kinase activation disrupts opioid receptor signaling. Proc Natl Acad Sci U S A. 2010;107:11608-13.

\section{Submit your next manuscript to BioMed Central and we will help you at every step:}

- We accept pre-submission inquiries

- Our selector tool helps you to find the most relevant journal

- We provide round the clock customer support

- Convenient online submission

- Thorough peer review

- Inclusion in PubMed and all major indexing services

- Maximum visibility for your research

Submit your manuscript at www.biomedcentral.com/submit 